\title{
PISCICULTURE ET RECHERCHES PISCICOLES EN HONGRIE
}

\author{
par J. WURTZ-ARLET
}

Chargée de Recherches ä I'I.N.R.A. Station d'Hydrobiologie continentale de l'aris

La Hongrie, c'est 9.300 .000 ha situés pour les deux tiers à moins de $200 \mathrm{~m}$ d'altitude, dominés par un massif montagneux $(1.015 \mathrm{~m})$. Le climat est continental $\left(-34^{\circ}\right.$ à $\left.+45^{\circ}\right)$ avec des années d'exceptionnelle sécheresse tournant à la catastrophe. Pourtant, c'est en Hongrie que l'on trouve le plus grand lac d'Europe, le lac Balaton, 60.500 ha, semblable à un vaste étang dont la profondeur est, en moyenne de $4 \mathrm{~m}$. Sa production atteint $1.400 \mathrm{t}$ environ : brèmes, brochets, carpes, sandres.

Les fleuves : Danube, Tisza, Koros, etc... couvrent 60.000 ha. Autrefois, des lots de $20 \mathrm{~km}$ étaient amodiés aux enchères à des pêcheurs professionnels. En 1934-1938, ils pêchaient $900 \mathrm{t}$ environ. Maintenant les coopératives ont fourni, en 1964, $2.970 \mathrm{t}$, plus $600 \mathrm{t}$ prises par des pêcheurs libres (brèmes, carpes, sterlets, silures).

La superficie des étangs et leur rendement suivent eux aussi une courbe ascendante :

$\begin{array}{lrr}1918 \ldots \ldots & 8.000 \text { ha } & 1.500 \mathrm{t} \\ 1938 \ldots \ldots & 10.000 \text { ha } & 4.000 \mathrm{t} \\ 1954 \ldots \ldots & 12.000 \text { ha } & 4.600 \mathrm{t} \\ 1961 \ldots \ldots \ldots & 20.000 \text { ha } & 16.000 \mathrm{t} \\ 1964 \ldots \ldots & 24.000 \text { ha } & 18.000 \mathrm{t}\end{array}$

Dans cette production, la Carpe occupe la plus grande place, $98 \%$, puis vient le Silure dont il est mis $83 \mathrm{t}$ sur le marché et $122 \mathrm{t}$ en alevins dans les divers plans d'eau. Les Hongrois consomment actuellement $2 \mathrm{~kg}$ de poissons d'eau douce par personne; le reste de la production part à l'exportation. Un projet portant sur 20.000 ha d'étangs nouveaux dans la Puszta d'Hortobay est en voie de réalisation.

Le domaine piscicole est exploité de trois façons différentes : fleuves et les lacs;

5.000 ha par vingt-deux coopératives agricoles exploitant les

- 10.000 ha par des fermes d'état agricoles et piscicoles;

- 9.000 ha par des " trusts " d'État uniquement piscicoles.

Le Bureau des Pêches du Ministère de l'Agriculture donne des directives techniques aux piscicultures et assure la répartition d'alevins 
de diverses espèces produits dans les établissements spécialisés comme la Pisciculture de Dinnyès. Les recherches piscicoles poursuivies en Hongrie sont largement encouragées par l'État et leurs résultats sont vulgarisés et mis à la portée des responsables des piscicultures.

\section{I. -- LA STATION EXPERIMENTALE DE SZARVAS.}

L'Institut de Recherches Piscicoles dépendant de la Faculté des Sciences Agricoles de Gödollö forme une vingtaine de chercheurs spécialisés en pisciculture chaque année et est fréquenté non seulement par des jeunes Hongrois, mais par des étudiants venus du monde entier. En plus des cours suivis à l'Institut, des étudiants font des visites ou des séjours à la Station expérimentale de Szarvas, qui est un centre de recherches agronomiques où le poisson est considéré comme une production agricole et où l'étang joue un rôle agronomique très important. En 1952, c'était seulement une ferme de 50 ha, station d'essais d'irrigation, où l'on construisit une dizaine de bassins pour utiliser l'eau amenée là par pompage. ('est en 1957 que le ministère donna les moyens d'en faire une Station de Recherches qui, maintenant, exploite 700 ha; mais si 350 ha sont des bassins et étangs permanents et 100 ha (le la terre en culture permanente, le reste est alternativement en eau ou en assec avec cultures.

L'ensemble des recherches est dirigé par Michael Szalai et son adjoint Janos Bakos, aidés par dix techniciens spécialisés et environ 200 employés. Le domaine est partagé en trois districts; autour des laboratoires, on trouve des étangs de petites dimensions où les recherches commencées en aquarium se poursuivent en bassins de $100 \mathrm{~m}^{2}$, puis dans 32 bassins de $1.500 \mathrm{~m}^{2}$ et 200 étangs inférieurs à deux hectares. A cinq kilomètres de là, quatre jeux de 16 bassins en étoile avec unique pêcherie centrale, présentent un aspect assez commode d'étangs expérimentaux. Enfin, dans Horwath Puzta, 400 ha de grands étangs, dont quatorze semblables de 8 ha, permettent les études agronomiques de l'amélioration du sol après trois années de mise en eau, notamment l'étude du rendement des diverses rotations.

La méthode des parcelles avec cinq répétitions a pu indiquer par exemple qu'en première année d'assec, la luzerne et le trèfle incarnat donnent le meilleur rendement avec $20 \%$ d'augmentation. points :

Les recherches à proprement parlé piscicoles portent sur plusieurs

- Sélection des carpes. Les souches proviennent de diverses piscicultures hongroises; comparaison de la croissance, de la composition chimique de la chair, de la fécondité, en rapport avec les croisements.

- Pathologie et parasitisme, culture de bactéries, élevage de parasites et essais de traitement.

- Mise au point de nourriture sèche et concentrée pour les carpes. La fabrication est faite sur place avec un matériel assez primitif.

- Analyse chimique des eaux, analyse du sol, mais aussi analyse 
de la chair des carpes durant tous les essais d'alimentation, de fertilisation. Les teneurs en protéines, en graisse et en eau de la chair dépendent de la nourriture et de la fréquence du nourrissage, de la fertilisation par engrais minéraux ou par fumure organique.

- Reproduction artificielle des poissons : brochets, sandres, carpes et silures, occupant successivement le magnifique laboratoire tout en faïence blanche avec bouteilles de Zug, bacs de réchauffement, d'éclosion, etc. Une source d'eau à $42^{\circ}$ est captée près du laboratoire et sert à réchauffer l'eau des bacs, aussi bien d'ailleurs que les bassins extérieurs de stockage en hiver.

Cette installation permet à M. Szalai d'envisager avec confiance la réalisation en 1968 de la reproduction artificielle des poissons herbivores,



Fig. 1. - Cohabilation des carpes et canards entre berge et mangeoires.

importés de Russie et de Chine. Les premiers alevins qu'il a reçus pèsent maintenant $2 \mathrm{~kg}$. Les expérimentations dans des bassins de $1.600 \mathrm{~m}^{2}$ en polyculture bien équilibrée, donnent à espérer un rendement possible de $1.700 \mathrm{~kg} / \mathrm{ha}$ tandis que les Myriophylle, Lemna, Wolfia, Potamogeton, ou même la luzerne fraîchement coupée, sont dévorés.

Enfin, la réalisation dont s'enorgueillit à juste titre la Station de Szarvas, est la mise au point d'un élevage rationnel combiné des carpes et des canards : à la rotation plus ou moins classique que l'on connaît dans bien des régions (2-4 ans d'étang -3 ans de culture), on a pu ajouter une production supplémentaire bénéfique à la fois à la phase piscicole et à la phase végétale : l'élevage de canards blancs de Pékin dans les étangs d'engraissement des carpes (K1 $\rightarrow \mathrm{K} 2$ ou K2 $\rightarrow \mathrm{K} 3$ ). On met à l'hectare $5.000 \mathrm{~K} 1$ et 160 canetons de trois semaines. Un hectare d'étang peut produire ainsi une tonne de poisson et deux tonnes de canards par an. En 1964, on a produit 160.000 canards. 
Les canetons, après une semaine en parquet, sortent en enclos pendant 10 à 12 jours, puis sont transportés sur les étangs vers 20 jours (à 24 jours quand la température de l'eau est à $6^{\circ}$ ). Les canards sont sacrifiés à 50-55 jours; ils pèsent de 2,5 à $2,7 \mathrm{~kg}$. La croissance est plus rapide qu'en parquet. Les mangeoires automatiques pleines de granulés sont placées à plusieurs mètres des berges. Des îles artificielles, flottantes, permettent aux canards de se reposer, mais ils ne disposent que d'un mètre carré pour 10 canards, ce qui les oblige à nager le plus souvent et la fumure est ainsi mieux répartie dans l'étang. Un kilogramme de canard produit environ $5 \mathrm{~kg}$ d'excrément, très riche en phosphore, azote et calcium. La quantité de granulés nécessaires pour faire un kilogramme d'accroissement dépend de la valeur nutritive des granulés et aussi des organismes vivants que le canard trouve dans l'étang. On compte de 2,5 à $4 \mathrm{~kg}$ à l'automne. Les pertes en canards, au cours de l'élevage à l'extéricur, sont faibles, environ 3 à $4 \%$. Quant aux poissons, ils ne subissent de ce voisinage aucun inconvénient mais, bien au contraire, l'étang est enrichi et le plancton très abondant explique l'augmentation de la productivité naturelle qui peut passer de $200 \mathrm{~kg}$ à 400 et même $600 \mathrm{~kg} / \mathrm{ha}$. La qualité de la chair des carpes, moins grasse, est meilleure. Le fond de l'étang subit également un enrichissement remarquable qui se traduit par une production très élevée en luzerne, trèfle, maïs et riz.

\section{II. - LA PISGIGULTURE DE DINNYES.}

La plus proche de Budapest et la plus récemment construite (1961), la Pisciculture de Dinnyès est spécialement conçue pour la production d'alevins. Le lac Velence (2.000 ha) eutrophe, riche en plancton, alimente par pompage l'ensemble de l'installation, c'est-à-dire 120 ha répartis ainsi : trois étangs de 15, 25 et 30 ha, deux de 8 ha, six de un hectare, six de 0,5 ha, 20 bassins de $175 \mathrm{~m}^{2}$. Un laboratoire de reproduction artificielle, construit en 1963, produit successivement plusieurs espèces d'alevins; au début du mois de février, on commence la fécondation artificielle des œufs de brochets et les alevins de 6 semaines sont vendus vers le $1^{\text {er }}$ avril; en 1965, la pisciculture a livré 3 millions de brochetons. Ensuite, on utilise le matériel pour la production artificielle de sandres, puis pour celle des carpes : en 1964, trente femelles ont reçu un traitement hypophysaire ; 14 seulement ont donné des œufs fertiles, produisant 2 millions d'alevins de trois semaines environ. Par la méthode naturelle en Dubisch la pisciculture a obtenu 5 millions d'alevins du même âge. Le transport de ces alevins vers les piscicultures est effectué par l'un des trente camions du Bureau des pêches sur lequel on charge dix bidons de 60 l contenant soit un million d'alevins de 20 jours, soit 200.000 alevins de 6 semaines.

C'est à Dinnyès qu'ont été livrés les premiers alevins de poissons herbivores ; en 1963, deux ingénieurs des pêches sont allés à Pékin étudier l'élevage de ces poissons et ont rapporté 54.000 sujets de 2 à $4 \mathrm{~cm}$. Actuellement (août 1965) ils pèsent près de $3 \mathrm{~kg}$. En juin 1964, la pisciculture ukrainienne de Krasnodar envoyait 600.000 alevins d'un jour 




Fig. 2. - Echantillonnage dans un étang de grossissement de deuxième été (Pisciculture de Dinnyes).



Fig. 3. - Un bel exemplaire d'Hypophtalmicthys molitrix de trois étés (Pisciculture de Dinnyes). 
qui pesaient, en octobre 1964,27 à $42 \mathrm{~g}$ et en août $1965,150 \mathrm{~g}$. En juin 1965, la même pisciculture de Krasnodar fournissait encore 500.000 alevins d'un jour. Le Directeur de la Station de Dinnyès A. Antalfi a effectué plusieurs pêches d'essais dans les étangs et j'ai pu voir des milliers d'alevins de 5 semaines pesant jusqu'à $5 \mathrm{~g}$ des espèces Ctenopharyngodon idella et Hypophtalmicthis molitrix.

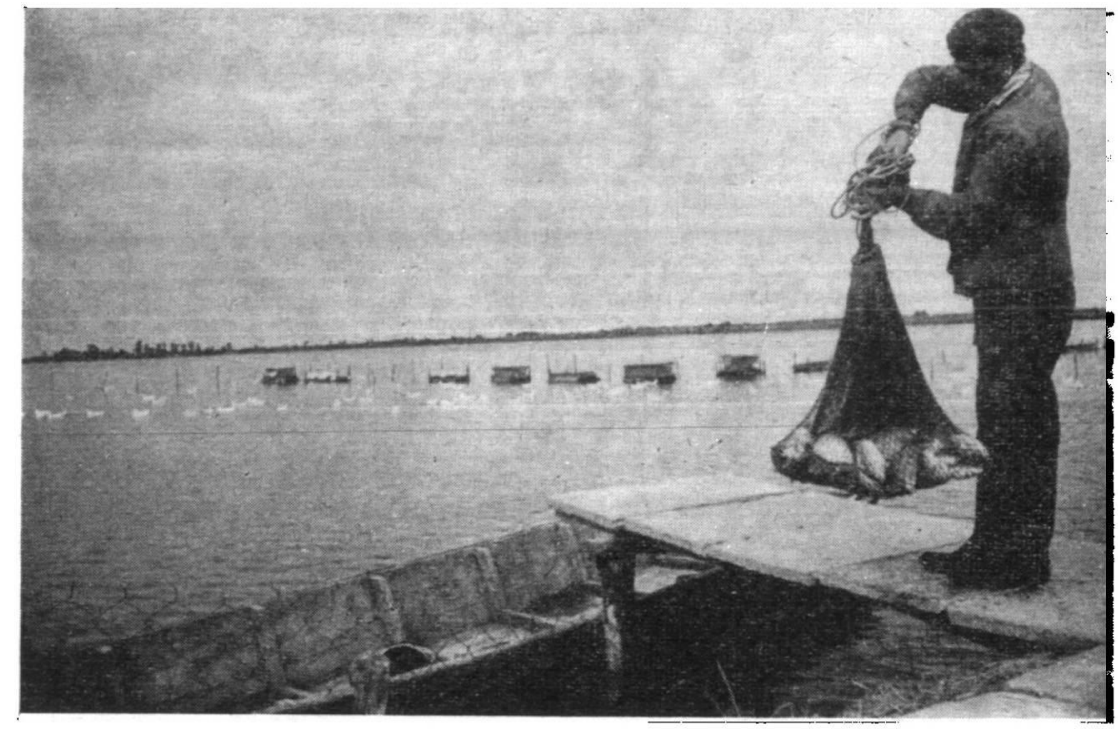

Fig. 4. - Pêche à l'épervier près d'un poste d'alimentation.

Ces poissons herbivores contrôlent parfaitement la végétation des étangs et un étang de 10 ha a été nettoyé complètement par quatre Ctenopharyngodon idella de deux kilogrammes introduits au printemps. Les alevins de Ctenopharyngodon et d'Hypophtalmicthis sont nourris pendant les premières semaines comme les alevins de brochets et de carpes avec du plancton du lac de Velence, filtré aux pompes. De la pisciculture de Dinnyès, nous espérons qu'un certain nombre d'exemplaires seront envoyés en France pour des expérimentations de contrôle de végétation en eau close.

\section{III. - LA COOPERATIVE DE PAKS.}

C'est l'une des 22 coopératives dont F. Bencze est responsable. Créée en 1945, elle est une des plus anciennes et comprend 2.000 ha de terre, 80 ha d'étangs et 3.000 ha de Danube. Quarante personnes s'occupent de l'exploitation piscicole etodu la prction totale s'élève à $200 \mathrm{t}$. 
Dans le Danube, on pêche, avec des grands bateaux qui traînent filets et nasses : silures, sandres, sterlets, barbeaux, chevesnes, perchessoleil, ablettes, pour moitié, et brèmes pour l'autre moitié.

Les huit étangs construits en 1958, près de Birito, sur sol sableux reçoivent de l'engrais (phosphate et urée) chaque semaine, du 1er avril au 1 er septembre. Ils sont empoissonnés avec des alevins de l'année ou d'un été ; carpes, silures, nourris 6 jours sur 7 avec : maïs, orge, lupin, pois, selon l'approvisionnement.

Une pêche d'essai m'a permis de constater la croissance des Carpes $\mathrm{K}_{1}$ pesant en avril 40 et $100 \mathrm{~g}$. Le 1 er août, elles pesaient 520 et $700 \mathrm{~g}$ en moyenne. On compte sur une production de $1.200 \mathrm{~kg} / \mathrm{ha}$.

La coopérative livre le poisson à des poissonneries et possède ses propres restaurants, sur le bord du Danube et en ville. Celui de Budapest est fort renommé ; on y déguste tous les poissons d'eau douce, et surtout la carpe, accommodés d'une vingtaine de façons, en écoutant un excellent orchestre gitan.

\section{IV. - LA PISGIGULTURE D'ÉtAT DE SZEGED.}

Neuf entreprises d'État sont spécialisées en pisciculture et celle de Szeged avec 1.600 ha est dirigée par M. Horwatr. Elle compte 16 étangs dont la superficie est environ 100 ha (deux atteignent 200 ha) et emploie 117 salariés. La production de 1.400 t est composée pour $95 \%$ de carpes marchandes ( $25 \%$ à l'exportation) et, pour le reste, de silures, tanches et sandres. Une conserverie absorbe une grande partie de la production.

Ainsi que me l'a exposé $M$. l'ingénieur Tasnadr, la pisciculture présente deux particularités. D'abord ses caractéristiques géologiques : elle est située au centre d'une cuvette de $860 \mathrm{~km}^{2}$ creusée par l'érosion glaciaire, comblée par les vents et le ruissellement. Le sol est formé de couches de carbonates $\left(\mathrm{CO}_{3} \mathrm{Na}_{2}, \mathrm{CO}_{3} \mathrm{Mg}, \mathrm{CO}_{3} \mathrm{Ca}\right)$ sur dix mètres d'épaisseur. C'est l'eau de la Tisza, amenée par un canal de $11 \mathrm{~km}$, qui alimente les étangs et l'analyse chimique montre une eau encore très carbonatée : pH 8,5. Extrait sec : 860 à $1.210 \mathrm{ppm}-\mathrm{Na}: 185$ à $200 \mathrm{ppm}-\mathrm{CO}_{3}:$ 500 à $600 \mathrm{ppm}$.

Autre particularité, le domaine est placé sur la grande voie des oiseaux migrateurs, dont 254 espèces passent et 196 espèces nichent. Malheureusement 3.500 couples de mouettes font des ravages parmi les alevins dont on estime à 3 millions la quantité mangée en 1964. Ce fait a poussé la pisciculture vers la production en laboratoire d'alevins de carpes. En effet, il reste seulement, à l'automne, $2 \%$ des alevins nés en étangs.

La production de carpes marchandes de trois étés pesant de 1 à $2 \mathrm{~kg}$ nécessite chaque année huit millions d'alevins de 8 jours, 2,5 millions de $K_{1}$, un million de $K_{2}$. Il y a un stock de 4.300 reproducteurs.

Le laboratoire de reproduction artificielle comporte, outre la chaudière de réchauffement de l'eau, 20 bouteilles de Zug, les bacs de préchauffage, et les bacs d'éclosion. Huit étangs d'un hectare sont aménagés pour 
recevoir les alevins de 8 jours. Pour donner un exemple de rendement de la méthode, en 1964, 41 femelles ont fourni 4,2 millions d'alevins de 8 jours, devenus 409.000 alevins de $30 \mathrm{~g}(12 \mathrm{t})$ à l'automne.

La pisciculture distribue $2.600 \mathrm{t}$ de nourriture par an, orge ou maïs. Huit kilomètres de voie étroite Decauwille permettent la circulation à longueur de journée des convois de grains et d'engrais tirés par des mules ou des chevaux. Le grain déversé dans des bacs en bordure des étangs trempe $24 \mathrm{~h}$ avant d'être réparti par bateaux aux points de nourrissage. Les poissons sont nourris 7 jours sur 7 .

C'est également chaque jour que l'on épand du fumier de porc liquide mélangé avec du superphosphate, du 1 er avril au 1 er août. Dans l'année, on répartit 12 à $14 \mathrm{t}$ de phosphate et $120 \mathrm{t}$ de fumier : cela représente à peu près 10 à $15 \mathrm{~kg} / \mathrm{jour} / \mathrm{ha}$ de fumier de porc. Les épandages sont arrêtés le $1^{\text {er }}$ août car ils provoquent une telle prolifération d'algues, en particulier des fleurs d'eau de Microcystis, que la teneur en oxygène devient critique au lever du jour (2,5 ppm) et le risque s'aggraverait avec les chaleurs du mois d'août. La pisciculture produit également des alevins de silures et en livre environ 250 à 300.000 d'un été $(5 \mathrm{~cm})$.

\section{V. - AUTRE ENTREPRISE D'ÉTAT : \\ LA PISGIGULTURE DE BIHARUGRA.}

Située près de la frontière roumaine, elle couvre 1.700 ha en 26 étangs sur lesquels sont combinées deux productions : poissons et canards à l'échelle industrielle. En effet, à $1.700 \mathrm{t}$ de carpes, tanches, sandres et silures, s'ajoutent $500 \mathrm{t}$ de canards, c'est-à-dire de 180.000 à 210.000 pièces produites par an dont s'occupent 26 personnes.

Les carpes, marchandes en trois années, sont nourries chaque jour avec de l'orge. La fertilisation est assurée d'une part par l'épandage de $100 \mathrm{~kg}$ de $\mathrm{P}_{2} \mathrm{O}_{5}$ à l'hectare et d'autre part par les excréments de canards qui sont élevés sur les étangs. Le fumier de canard est beaucoup plus riche que celui de porc, généralement utilisé en pisciculture en Hongrie.

De mars à octobre, dix machines incubatrices de 8.000 œufs assurent l'éclosion des œufs de 2.800 canes reproductrices, blanches de Pékin. La vie des canards, de l'œuf au marché, dure 51 jours : le canard vendu vivant pèse alors $2,400 \mathrm{~kg}$. Les canetons élevés en parquet pendant 3 à 4 semaines sont ensuite lachés sur les étangs empoissonnés de $C_{1}$ ou $C_{2}$. A 7 jours, ils pèsent $180-200 \mathrm{~g}$; à 14 jours, $350-370 \mathrm{~g}$ et à 4 semaines : $500 \mathrm{~g}$. Ils trouvent leur nourriture dans les mangeoires automatiques placées dans l'étang. Le prix de revient est évalué à 16 forints/kg et le canard est vendu vivant 20 forints $/ \mathrm{kg}$. Le kilogramme de carpe vaut 14 à 16 forints. Le directeur L. Kosma envisage d'augmenter encore la superficie des étangs et la production de canards dont le principal débouché est l'exportation. 
J'ai également rendu une visite d'amitié à Mme Olga Sebesyten qui, malgré sa retraite, travaille encore dans l'Institut Biologique de l'Académie des Sciences de Hongrie, à Tibany, sur les bords du lac Balaton. M. le Professeur Woynarovich m'a accompagnée au cours de quelques déplacements, en particulier pour visiter l'Institut de Zoologie à l'Université Kossuth à Debrecen.

Mon voyage en Hongrie a été dominé par le souci de mieux connaître la biologie, les habitudes alimentaires et les possibilités d'adaptation des poissons herbivores d'origine chinoise aux conditions du climat et des étangs européens. C'est pourquoi le moment le plus passionnant de mon séjour en Hongrie a été l'heure du repas de Ctenopharyngodon idella à l'Aquarium du jardin zoologique de Budapest. L'Ingénieur B. Penzes me laissa composer le menu (Chara sp., Myriophyllum, Potamogeton perfoliatus, Cladophora sp.) des 10 exemplaires de Ctenopharyngodon âgés de 14 mois, pesant environ $150 \mathrm{~g}$ et mesurant $20 \mathrm{~cm}$. En un quart d'heure, ils ont mangé à peu près $500 \mathrm{~g}$ de plante, en commençant par les Chara, avec grand appétit. La réputation de ce poisson comme herbivore était bien une réalité et je trouvais à ce spectacle le meilleur encouragement pour entreprendre une expérimentation, certes coûteuse, dans des eaux closes françaises où le faucardage et le désherbage chimique sont impossibles. Les observations que j'ai faites dans les diverses piscicultures hongroises m'ont permis de mieux comprendre les possibilités et les limites d'utilisation des trois espèces chinoises (1) dans les eaux françaises.

Je ne saurais terminer ce compte rendu sans insister sur la courtoisie, la patience, la gentillesse et je peux dire l'amitié qui a présidé à toutes mes visites avec mes collègues hongrois. Aussi bien les directeurs, les ingénieurs, les interprètes, les chauffeurs, chacun a eu à cœur de faciliter et de rendre plus fructueux chaque déplacement, chaque rencontre.

Je rendrai particulièrement hommage à $M$. le Directeur du Bureau des Pêches, M. Ribiansky, auquel je dois l'organisation de la tournée d'étude et qui, sans pouvoir m'accompagner chaque jour, a bien voulu déléguer auprès de moi ses meilleurs collaborateurs.

Nota. - Une visite des installations piscicoles hongroises est organiscé du $1^{\text {er }}$ au 8 mai 1967.

Prix du voyage Paris-Paris : 995 F (Billet avion, pension complète et visiles techniques de six piscicultures).

Pour y participer, s'adresser sans délai à Mme Wuntz-ArLET, 14, avenue de Saint-Mandé, 75 - Paris $\left(12^{e}\right)$.

(1) N. D. L. R. - Un article de MM. Penzes et Tolg, traitant du réginte alimentaire de ces poissons, paraîtra dans le prochain Bulletin. 